\title{
How an Individual's Self-Construals, Cosmopolitan and Local Orientation Affect the Impact of the Four Self-Congruity Types on Brand Attitude
}

\author{
Hector Gonzalez-Jimenez, Fernando Fastoso, and Kyoko Fukukawa
}

\begin{abstract}
Recent research questions the universality of the self-congruity effect on brand perceptions and highlights the need for further clarifying the boundary conditions of self-congruity theory. Current evidence suggests that it is individual characteristics that determine the superiority of branding strategies focused on appealing to a consumers' actual versus ideal self-congruity. This study extends that research by modelling how four individual characteristics of value for cross-cultural market segmentation purposes (an individual's level of independence, interdependence, and their cosmopolitan and local orientations) affect which of the four self-congruity types (actual, ideal, social, and social ideal) has the strongest impact on brand perceptions. Empirically, the validity of the conceptual framework is tested using survey data from non-student samples in the USA and India. Findings show that an individual's level of independence and interdependence, cosmopolitan and local orientation determine which self-congruity type has the strongest effect on brand attitude. Specifically, the results show that for locals and interdependents, actual self-congruity has the strongest effect on brand attitude. In contrast, for cosmopolitans and independents, ideal self-congruity has the strongest effect on brand attitude. Interestingly, overall actual and ideal self-congruity types have the strongest effect on brand attitude for the consumer types tested. Moreover, the findings are partially validated in both countries and suggest that other factors pertaining to the USA and India may influence the self-congruity effect. The study offers practitioners with insights on which self-concept type they should try to match in their brand communications to elicit most positive brand attitude among consumers depending on their individual characteristics.
\end{abstract}

H. Gonzalez-Jimenez $(\bowtie) \bullet$ F. Fastoso

University of York, Heslington, UK

e-mail: hgj503@york.ac.uk; fernando.fastoso@york.ac.uk

K. Fukukawa

Bradford University, Bradford, UK

e-mail: K.Fukukawa@bradford.ac.uk 